\title{
The Effects of The Royal Jelly On Dry Mouth Sensation With Normal Saliva Function: A Double- Blind, Placebo-Controlled, Cross-Over Trial Clinical Study
}

Yumi Mochizuki ( $\square$ mochizuki.osur@tmd.ac.jp )

Tokyo Medical and Dental University

Fumihiko Tushima

Tokyo Medical and Dental University

\section{Yuji Kabasawa}

Tokyo Medical and Dental University

Hiroyuki Harada

Tokyo Medical and Dental University

\section{Research Article}

Keywords: royal Jelly, dry mouth sensation, normal salivary function, burning mouth syndrome ,a doubleblind, placebo-controlled, cross-over trial clinical study

Posted Date: December 30th, 2021

DOI: https://doi.org/10.21203/rs.3.rs-969279/v1

License: (1) This work is licensed under a Creative Commons Attribution 4.0 International License.

Read Full License 


\section{Abstract}

Background: Because there are no standardized treatment methods for dry mouth sensation with normal salivary function, cases of dry mouth sensation continually suffer. The aim of this clinical study was to evaluate the effects of a royal jelly tablets on dry mouth sensation, in patients with normal salivary function.

Methods: This double-blind randomized clinical trial was carried out in the Department of Oral and Maxillofacial Surgery, Tokyo Medical and Dental University Hospital between November, 2019 and April, 2020. This clinical trial was randomized, double-blind, and of a crossover design, with a four-week washout period. Each participant received either $800 \mathrm{mg}$ of royal jelly tablets or placebo, daily for 12 weeks.

Results: Differences in VAS scores between royal jelly tablets and placebo were evaluated at 4 and 12 weeks, and were significant. Differences in HADS and GHQ12 scores between those exposed to royal jelly tablets and placebo, at the evaluation period of 12 weeks, were statistically significant. A statistically significant decrease in HADS scores in the royal jelly tablet group, was observed over time.

Conclusions: These findings show that daily consumption of royal jelly (800 mg) for 12 weeks was effective in alleviating dry mouth sensation with normal saliva function.

Clinical trial registration numbers and the full date of first registration: ClinicalTrials.gov (jRCTs031190067); 02/08/2019

\section{Background}

Many cases of dry mouth sensation with the normal salivary function are diagnosed as non-abnormality. Because there are no standardized treatment methods for dry mouth sensation with normal salivary function, several people continue to suffer from dry mouth sensation.

Recently, it was reported that the anxiety and depression score of patients who suffered from subjective dry mouth sensation, in spite of unstimulated salivary flow $(>0.1 \mathrm{ml} / \mathrm{min})$, was statistically significantly higher than that of the patients with unstimulated salivary flow $(>0.1 \mathrm{ml} / \mathrm{min})$, with no subjective dry mouth sensation [1, 2]. Dry mouth sensation with normal salivary function is considered to have a statistically significant relationship with psychological disorders such as depression, trait anxiety, and perceived stress $[1,2]$.

Royal jelly, a viscous jelly-like substance, is produced by the mandibular and hypopharyngeal glands of honeybees (Apis mellifera). It consists of water (50-60\%), proteins (18\%), carbohydrates (15\%), lipids (3$6 \%)$, mineral salts (1.5\%), and vitamins and reportedly, non-cytotoxic [3]. It could be easily-acquired at stores and widely used as a nutritional natural supplement. Recently, royal jelly supplementation has been reported to have beneficial effects on menopausal symptoms such as anxiety, backache, neck 
stiffness and headache $[4,5]$. However, there are no studies on royal jelly supplementation for dry mouth sensation with normal salivary function. We hypothesized royal jelly supplementation is effective for dry mouth sensation with normal saliva function.

The purpose of this clinical study is to evaluate and research the effects of a royal jelly-containing tablet, on dry mouth sensation with normal saliva function.

\section{Methods}

This clinical trial was carried out according to the Declaration of Helsinki. Ethical approval and registration of this clinical study were approved by the Ethical Committee for Human Research at Tokyo Medical and Dental University (No.D2019-021) and Tokyo Medical and Dental University Certified Clinical Research Review Board (NR2019-002). The trial is registered with Japan Registry of Clinical Trials.gov protocol registration system, ID jRCTs031190067. The full date of first registration was $02 / 08 / 2019$. All patients provided written informed consent before participating.

\section{Subjects}

The study population comprised of adults presenting with the chief complaint of dry mouth sensation and satisfy all of the following inclusion criteria: 1) age of at least 20 years and can visit our department and provide written consent of participation; 2) unstimulated salivary flow $₫ 0.1 \mathrm{~mL} / \mathrm{min}$ in the saliva secretion test; 3 ) those who do not have autoimmune diseases including Sjogren's syndrome or salivary gland disease; and 4) those who did not take royal jelly products at the start of this study. The exclusion criteria were as follows: 1) adults who developed allergies due to past drug or food consumption and 2) those who have a history of asthma.

The data were collected in at our Department of Oral and Maxillofacial Surgery, Tokyo Medical and Dental University Hospital.

\section{Trial design}

This clinical trial was a randomized, double-blind and crossover design with a four-week washout period. We administered the enzyme-treated royal jelly and the placebo tablet. Each royal jelly tablet contained $400 \mathrm{mg}$ of enzyme-treated royal jelly powder. The enzyme-treated royal jelly (lot.YRP-M-190315-2) was standardized to contain 3.5\% 10-hydroxy-trans-2-decenoic acid and 0.6\% 10-hydroxydecanoic acid was obtained from Yamada Bee Company, Inc. (Okayama, Japan).

The powdered enzyme-treated royal jelly and the placebo tablet were of similar size, shape, and color, and their packaging was labeled as $\mathrm{A}$ or $\mathrm{B}$. The researchers and the participants of this research are not known whether enzyme-treated royal jelly is labeled as A or B. If initially package was labeled as A, the packages labeled as $B$ were given after the washout period. Persons without personal relationships with the researchers, randomly assigned the subjects into two groups using a computer-generated random number. Every participant was assigned randomly to group 1 or 2 (description below), based on 
randomization codes, which were selected by a computer using dynamic allocation (with a balanced marginal distribution algorithm). Subjects assignments were held in a sealed file and only decoded at the end of the study period.

Group 1: Enzyme-treated royal jelly tablets were given at a dose of $800 \mathrm{mg} /$ day (1 tablet $(400 \mathrm{mg}$ ) $\times \times 2$ tab /day) were administered for 12 weeks followed by a four-week wash-out period. The placebo treatments were administered for another 12 weeks.

Group 2: The placebo treatment was administered for 12 weeks followed by a four-week wash-out period. Participants were then administered enzyme-treated royal jelly at a dose of $800 \mathrm{mg} /$ day (2 tablets) were administered for another 12 weeks. The protocol is demonstrated in Fig. 1.

The sample size was estimated using an effect size in the range of $d=0.7$, two-sided test with $a=0.05$, and a power of 0.8 . The results indicated a required sample size of 11 subjects for a crossover design. To allow for dropouts, we planned to include 15 randomized patients.

\section{Primary outcome measures}

Evaluation of objective dry-mouth feeling was by VAS scale, with 100 for dryness and 0 for no dryness. Psychological status was evaluated by the Japanese version of Hospital Anxiety and Depression Scale (HADS) [6].

The Japanese version of HADS evaluated two subscales of anxiety and depression. Each response was scored on a scale from 0 to 3 and summarized the score of 7 questions. Totally, subscale of anxiety and depression was scored from 0 to 21. According to Zigmond et al. [6], scores of $0-7$ represent a non-case of psychiatric morbidity, scores of 8-10 indicate a doubtful or borderline case, and scores of 11-21 indicate a definite case.

Psychological distress and social dysfunction were evaluated by the Japanese version of the General Health Questionnaire 12 (GHQ12) [7]. The GHQ12 evaluates two factors of psychological distress and social dysfunction. Each response is scored on a scale from 0 to 1 and summarized the score of 12 questions. If the score is 4 or more, psychological distress and social dysfunction is judged. Higher scores are indicative of a worsened status of psychological distress and social dysfunction. The reliability and validity of each questionnaire has been scientifically verified.

\section{Analyses}

The scores from the VAS, HADS, and GHQ 12 at the beginning of taking placebo or enzyme-treated royal jelly was used as the baseline data. We calculated the difference in the scores over time, by the following formula: each score of placebo or enzyme-treated royal jelly at $4 / 8 / 12$ weeks - each score at the stared period of placebo or enzyme-treated royal jelly.

The differences in VAS scales, HADS scores and GHQ12 scores between enzyme-treated royal jelly and placebo over-time were analyzed by repeated measure ANOVA and post-hoc paired $t$-tests. 
The differences at each examination point were analyzed with $t$-tests. For all the analyses, a $5 \%$ significance level was used to determine statistical significance. Statistical analysis was performed using the software program Statistical Package of Social Science (SPSS 25.0J, IBM, Armonk, NY).

\section{Results}

\section{Demographic characteristics}

Two men and 13 women participated in this study. The median age was 72.0 years old (range from 3178 years old). Among 15 participants, one was lost due to lack of contacting us. Finally, one man and 13 women completed the study. The compliance rate among all participants was $100 \%$. We did not observe any allergic reactions or abnormal side effects. After the end of research, two women refused to use their data for our research. Finally, we analyzed the research data of 12 cases.

\section{VAS scales scores}

A summary of the VAS results is shown in Fig. 2. There were no significant differences in VAS scale scores of placebo ( $d f=1, F=0.333, p=0.570)$ and in VAS scale scores of enzyme-treated royal jelly ( $d f=$ $2, F=1.243, p=0.308$ ) (Fig. 2).

Differences in VAS scores between enzyme-treated royal jelly and placebo at the evaluation period of 4 weeks ( $d f=1, F=4.717, p=0.041)$ and 12 weeks $(d f=1, F=4.636, p=0.043$ ) were significant, however, at 8 weeks differences in VAS scores between enzyme-treated royal jelly and placebo were not statistically significant ( $d f=1, F=0.018, p=0.896)$. There was no interaction between the evaluation periods and treatment ( $d f=2, F=2.508, p=0.093)$.

\section{HADS score}

A result summary for HADS score is provided in Fig. 3. There were no significant differences in HADS scores of placebo over time ( $d f=2, F=3.253, p=0.058)$. On the other hand, there was a statisticallysignificant decrease in HADS scores of enzyme-treated royal jelly, over time $(d f=2, F=8.789, p=0.002)$. Statistically significant differences in HADS scores of enzyme-treated royal jelly between the evaluation period of 4 weeks and 8 weeks $(p<0.001)$, between the evaluation period of 4 weeks and 12 weeks ( $p=$ $0.015)$ and between the evaluation period of 8 weeks and 12 weeks $(p<0.001)$, were observed (Fig. 2$)$. Differences in HADS scores between enzyme-treated royal jelly and placebo, at the evaluation period of 4 weeks ( $d f=1, F=2.097, p=0.162)$ and 8 weeks $(d f=1, F=0.096, p=0.760)$ were not significant, however, at 12 weeks, were statistically significant $(d f=1, F=4.754, p=0.040)$. There was a significant interaction between the evaluation periods and treatment $(d f=2, F=6.152, p=0.008)$.

\section{GHQ12 score}

The result summary for GHQ12 score is shown in Fig. 4. There were no significant differences in GHQ12 scores of placebo over time ( $d f=2, F=1.777, p=0.191)$ and GHQ12 scores of enzyme-treated royal jelly 
over time ( $d f=2, F=3.880, p=0.072)$ (Fig. 3). Differences in GHQ12 scores between enzyme-treated royal jelly and placebo at the evaluation period of 4 weeks $(\mathrm{df}=1, \mathrm{~F}=0.390, \mathrm{p}=0.539)$ and 8 weeks $(\mathrm{df}=$ $1, F=1.690, p=0.207$ ) were not significant, however, at 12 weeks, differences in GHQ12 scores between enzyme-treated royal jelly and placebo, were statistically significant $(d f=1, F=4.468, p=0.046)$. There was a significant interaction between the evaluation periods and treatment $(d f=2, F=3.925, p=0.027)$.

\section{Discussion}

Sharif et al. reported that daily consumption of oral royal jelly $(1000 \mathrm{mg})$ for eight weeks was effective in alleviating menopausal symptoms [5].

There were no reports on the effectiveness for dry mouth sensation with normal saliva function. This is the first report on the effects of the royal jelly on dry mouth sensation with normal saliva function by double-blind, randomized, placebo controlled clinical trial.

Bergdahl et al. suggested that although there are many unknown factors, depression, stress, and anxiety have complex interactions with subjective dry mouth sensation with normal salivary function [2].

In our results, HADS scale and GHQ12 scale improved over time with royal jelly supplements. It is considered that royal jelly supplements are effective for depression and anxiety associated with the subjective dry mouth sensation. Both scales significantly improved 12 weeks after the administration of royal jelly tablets, in comparisons with placebo administration. This result suggests that oral administration of royal jelly tablets at 12 weeks may be effective for treating dry mouth sensation.

Although VAS scale at the evaluation period of 4 weeks and 12 weeks insisted that the administration of royal jelly tablet significantly improved dry mouth sensation, VAS scales over time were unstable. The instability of the VAS scale over time may be due to oral symptoms caused by depression and anxiety, outside of dry mouth sensation.

Recently, burning mouth syndrome, which is defined as a burning sensation or as pain in the oral mucosa, has been reported [8-11]. This syndrome has an etiologically complex relationship with pathophysiological factors - especially depression and anxiety $[8,10]$. Dry mouth sensation with normal salivary function has been considered as one of the symptoms of burning mouth syndrome [9-12].

The participants of this study could potentially have burning mouth syndrome. Other uncomfortable oral sensations from burning mouth intricately effect on the VAS scale analysis and the VAS scale cannot accurately detect subjective dry mouth sensation. However, this study only targeted those who suffered from dry mouth with normal salivary function. Whether or not the participants had potential burning mouth syndrome was not a screening item for study inclusion and was unknown.

For dry mouth sensation with normal salivary function, standard treatment and medication are not established [8]. Royal jelly contains a unique component, 10-hydroxy-trans-2-decenoic acid (10H2DA), an unsaturated fatty acid [13]. 10H2DA represents one of the main bioactive components of royal jelly and 
constitutes the vast majority of the royal jelly lipid content ( 0.75 to $3.39 \%)$, and is one of the main bioactive components $[14,15]$.

Ito et al. reported in animal models that $10 \mathrm{H} 2 \mathrm{DA}$ was effective in improving the stress-induced symptoms of depression and anxiety [13].

In general, double-blind, randomized, placebo controlled clinical trials could provide more scientific evidence [10]. In the clinical trials for burning mouth syndrome there were few reports of long-term followup data beyond 2 months [10]. Our study provides important information for further detailed studies of dry mouth sensation with normal saliva function.

Our study revealed that the royal jelly could consumption ( $800 \mathrm{mg}$ dairy) for 12 weeks is effective for alleviating psychological stress (anxiety and depression) and social activity disorder in patients who suffered from subjective dry mouth sensation with normal saliva function. Further developmental studies are needed in the future.

Our study targeted a limited number of participants and those who suffered from only dry mouth sensation flow, and didn't include patients with burning mouth syndrome. The treatment of burning mouth syndrome is considered a difficult task for oral health care professionals [12]. Further developmental studies assessing the effects of royal jelly on burning mouth syndrome, induced by depression and anxiety, are expected in the future.

\section{Conclusions}

Our study reveals that 12 weeks of royal jelly tablets (800 mg dairy) administration is effective for psychological stress (anxiety and depression) in patients who suffer from subjective dry mouth sensation with normal saliva function.

\section{Abbreviations}

HADS Hospital Anxiety and Depression Scale

GHQ12 General Health Questionnaire 12

10H2DA 10-hydroxy-trans-2-decenoic acid

\section{Declarations}

\section{Ethics approval and consent to participate}

Ethical approval and registration of this clinical study was approved by the Ethical Committee for Human Research at Tokyo Medical and Dental University (No.D2019-021) and Tokyo Medical and Dental University Certified Clinical Research Review Board (NR2019-002). The trial is registered with Japan 
Registry of Clinical Trials.gov protocol registration system, ID jRCTs031190067. All patients provided written informed consent before participation.

Informed consent was obtained from all subjects involved in the study.

\section{Consent for publication}

We obtained consent for publication from the participants and all co-authors.

\section{Availability of data and materials}

The data that support the findings of this study are available from the corresponding author, upon reasonable request.

\section{Competing interests}

The authors declare that they have no competing interests.

\section{Funding}

This research was supported by the Yamada Research Grant under the Yamada Bee Company, Inc.

\section{Authors' contributions}

Conceptualization, methodology, formal analysis, investigation, YM; data curation, YM and AK; writingoriginal draft preparation, $\mathrm{YM}$; writing- review and editing $\mathrm{YM}, \mathrm{FT}$ and $\mathrm{HH}$; supervision, $\mathrm{YM}$ and $\mathrm{YK}$; project administration, YM; funding acquisition, YM. All authors have read and agreed to the published version of the manuscript. I removed punctuation signs after each authors' initials to ensure consistency with the above example.

\section{Acknowledgements}

The authors are grateful to Associate prof. Yoshiaki Hanzawa and Mariko Man (Department of Medical Innovation Promotion Center, Tokyo Medical and Dental University) (for their skillful technical advice throughout the study.

\section{References}

1. Gholami N, Sabzvari BH, Razzaghi A, Salah S. Effect of stress, anxiety and depression on unstimulated salivary flow rate and xerostomia. J Dent Res Dent Clin Dent Prospects. 2017;11:24752.

2. Bergdahl M, Bergdahl J. Burning mouth syndrome: prevalence and associated factors. J Oral Pathol Med. 1999;28:350-4. 
3. Buttstedt A, Moritz RFA, Erler S. More than royal food - Major royal jelly protein genes in sexuals and workers of the honeybee Apis mellifera. Front Zool. 2013;10:1-0.

4. Asama T, Matsuzaki H, Fukushima S, Tatefuji T, Hashimoto K, Takeda T. Royal jelly supplementation improves menopausal symptoms such as backache, low back pain, and anxiety in postmenopausal Japanese women. Evid Based Complement Alternat Med. 2018;2018.

5. Sharif SN, Darsareh F. Effect of royal jelly on menopausal symptoms: A randomized placebocontrolled clinical trial. Complement Ther Clin Pract. 2019;37:47-50.

6. Zigmond AS, Snaith RP. The hospital anxiety and depression scale. Acta Psychiatr Scand. 1983;67:361-70.

7. Doi Y, Minowa M. Factor structure of the 12-item General Health Questionnaire in the Japanese general adult population. Psychiatry Clin Neurosci. 2003;57:379-83.

8. Borahan M, Pekiner F, Atalay T. Evaluation of Effects of the Psychological Factors on Saliva. Journal of Marmara University Institute of Health Sciences. 2012;2:S8-14.

9. Feller L, Fourie J, Bouckaert M, Khammissa RAG, Ballyram R, Lemmer J. Burning mouth syndrome: Aetiopathogenesis and principles of management. Pain Res Manag. 2017;2017

10. Liu YF, Kim Y, Yoo T, Han P, Inman JC. Burning mouth syndrome: a systematic review of treatments. Oral Dis. 2018;24:325-34.

11. Petruzzi M, Lauritano D, De Benedittis M, Baldoni M, Serpico R. Systemic capsaicin for burning mouth syndrome: short-term results of a pilot study. J Oral Pathol Med. 2004;33:111-4.

12. Aravindhan R, Vidyalakshmi S, Kumar MS, Satheesh C, Balasubramanium AM, Prasad VS. Burning mouth syndrome: A review on its diagnostic and therapeutic approach. J Pharm Bioallied Sci. 2014;6:S21-S25.

13. Ito $S$, Nitta Y, Fukumitsu H, Soumiya H, Ikeno K, Nakamura T, et al. Antidepressant-like activity of 10hydroxy-trans-2-decenoic acid, a unique unsaturated fatty acid of royal jelly, in stress-inducible depression-like mouse model. Evid Based Complement Alternat Med. 2012;2012.

14. Kocot J, Kielczykowska M, Luchowska-Kocot D, Kurzepa J, Musik I. Antioxidant potential of propolis, bee pollen, and royal jelly: possible medical application. Oxid Med Cell Longev. 2018;2018.

15. Ali AM, Hendawy AO. Royal Jelly Acid, 10-Hydroxy-Trans-2-Decenoic Acid, for Psychiatric and Neurological Disorders. How helpful could it be?!. Edelweiss Food Sci Tech. 2019;1:1-4.

\section{Figures}

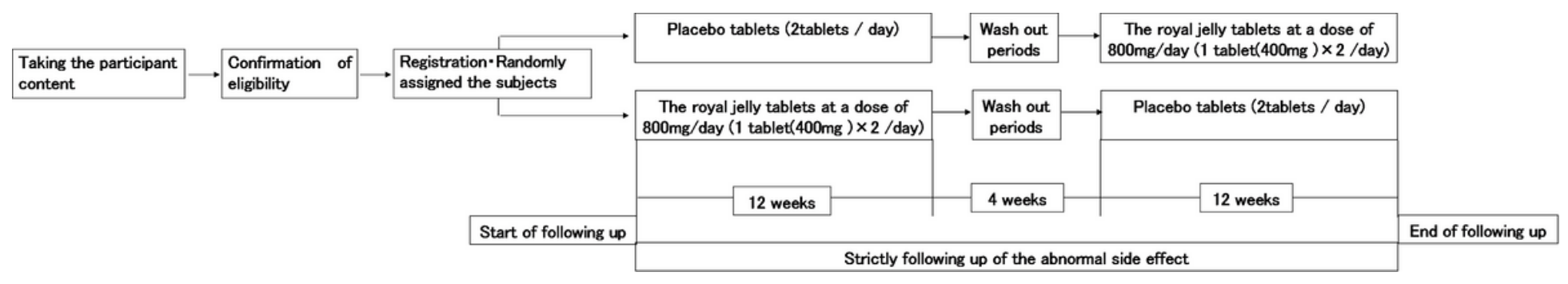


Figure 1

The administration protocol used in this study

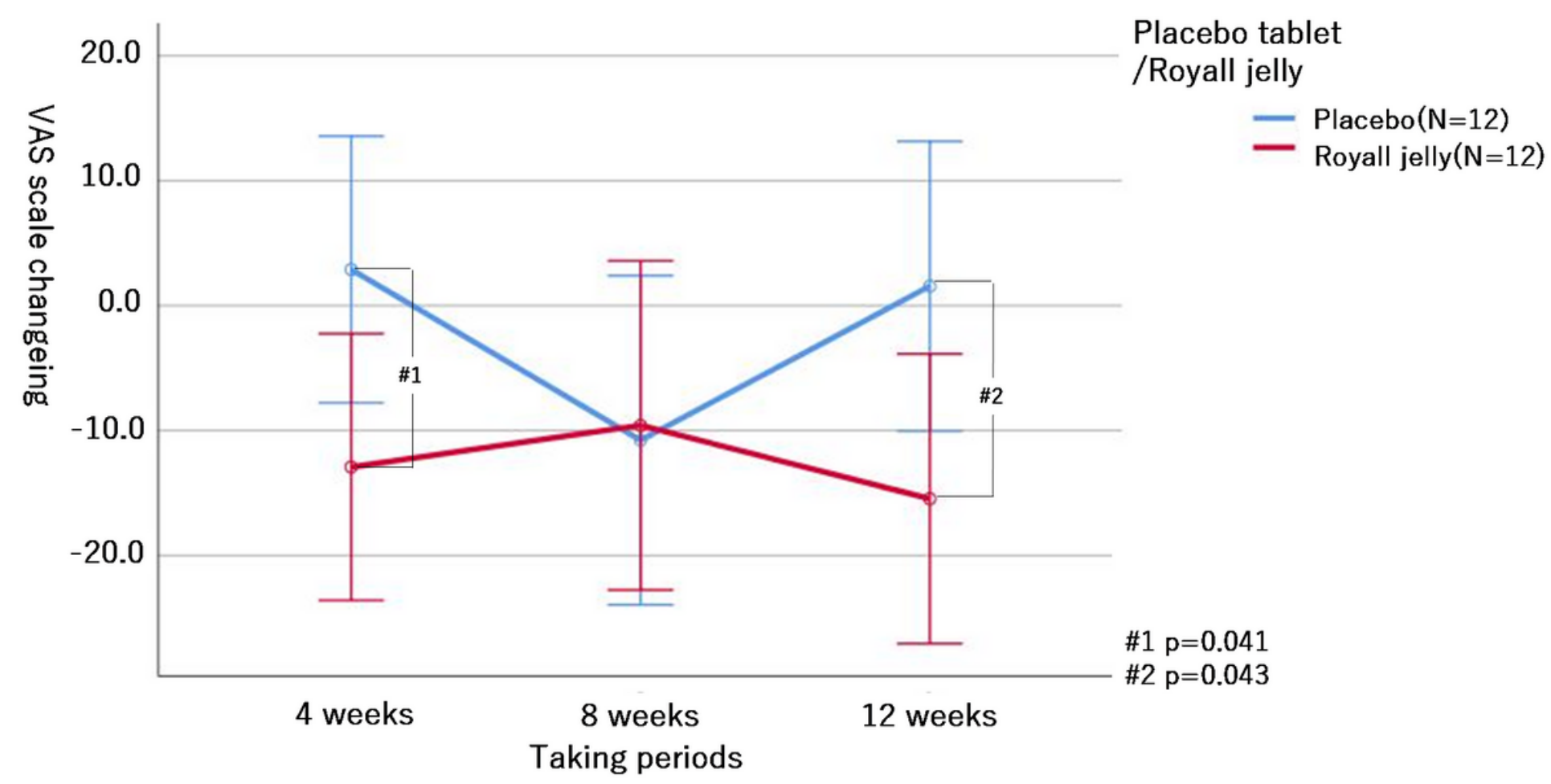

Figure 2

The changing trend of VAS scales score over time 


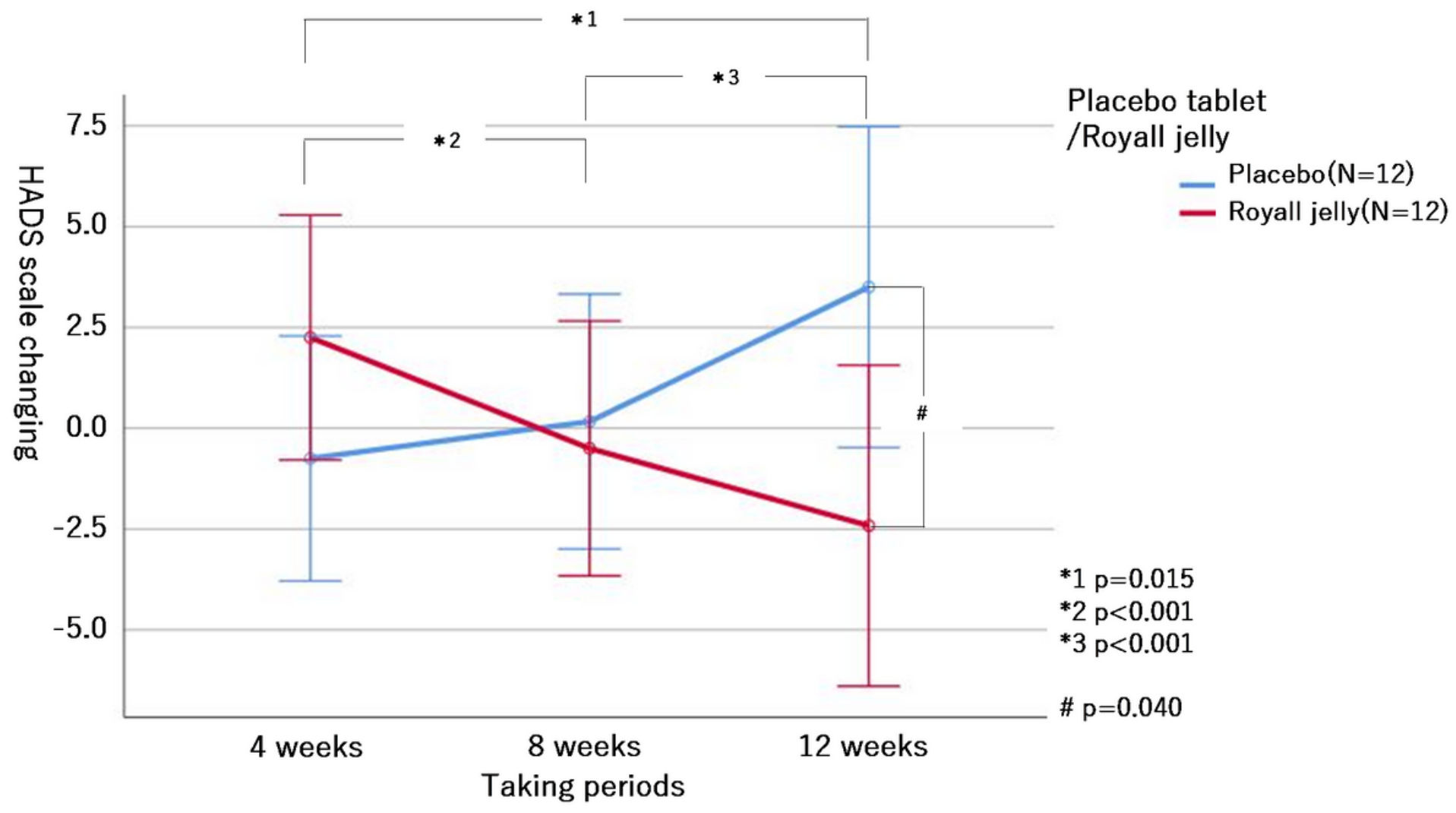

Figure 3

The changing trend of HADS scores over time 


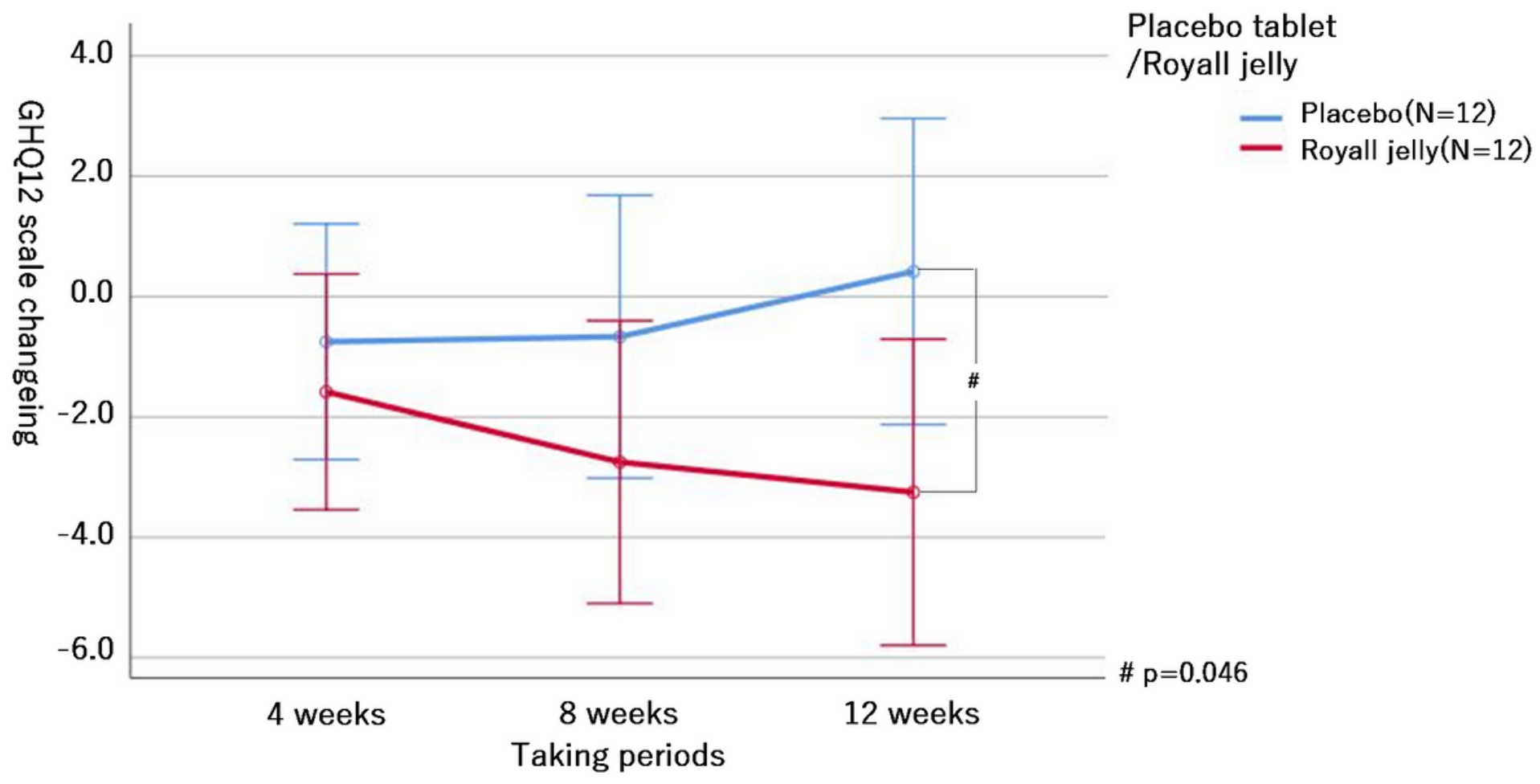

Figure 4

The changing trend of GHQ12 scores over time 